\title{
Prevalencia de trastornos psiquiátricos en pacientes ingresados por el Servicio de Psiquiatría en el Módulo Penitenciario del H.U.M.S.
}

\author{
Calvo Estopiñán Pa, Soler González C ${ }^{b}$, Día Sahún JCc, Ventura Faci T ${ }^{d}$
}

2a,b,c,d Servicio de Psiquiatría. Hospital Universitario Miguel Servet. Zaragoza.

\section{RESUMEN}

Introducción: Estudios epidemiológicos recientes destacan el aumento de prevalencia de trastornos psiquiátricos en presos. El objetivo del presente estudio es determinar los trastornos psiquiátricos principales y secundarios más prevalentes, datos sociodemográficos y estancia media, de los pacientes ingresados en el Módulo Penitenciario de un hospital general a cargo del Servicio de Psiquiatría.

Material y métodos: Estudio descriptivo transversal retrospectivo. La muestra está compuesta por los pacientes ingresados en el Módulo Penitenciario a cargo de Psiquiatría durante 5 años, siendo n=136.

Resultados: El 90,4\% fueron hombres y el 9,6\% mujeres. La edad media fue de 34,18 años. Los diagnósticos psiquiátricos principales más prevalentes fueron: Tr. Personalidad 22\%, Tr. Esquizofrénicos 16,3\%. Como diagnósticos psiquiátricos secundarios más prevalentes encontramos: Tr. Personalidad 11,9\%, Abuso de drogas 10,7\%.

Conclusiones: El diagnóstico psiquiátrico principal y secundario más prevalente fue el trastorno de personalidad. Se documentó la existencia de una alta comorbilidad con el abuso-dependencia de tóxicos. Actualmente las prisiones carecen de equipos multidisciplinares en Salud Mental, con lo que en muchas ocasiones se hace necesario el traslado del preso al hospital con la carga asistencial y el incremento de costes que esto supone.

Palabras clave: Trastornos mentales; trastorno de personalidad antisocial; trastorno relacionado con sustancias; prisiones.

\section{PREVALENCE OF PSYCHIATRIC DISORDERS ON PATIENTS ADMISSED BY THE PSYCHIATRIC SERVICE IN THE SECURITY AREA OF H.U.M.S. (University Hospital "Miguel Servet”)}

\begin{abstract}
Introduction: recent epidemiological studies highlight an increase in the prevalence of psychiatric disorders amongst prison inmates. The objective of this study to determine the most prevalent primary and secondary psychiatric disorders, socio-demographic data and average stay period amongst patients admitted to the Prison Unit of a general hospital as charges of the Psychiatric Service.

Materials and Methods: retrospective cross-sectional descriptive study. The sample group consisted of a number of patients admitted into the Prison Unit as charges of the Psychiatric Service during a five year period $(\mathrm{n}=136)$.

Results: $90,4 \%$ were men and 9,6\% were women. The average age was 34,18 years. The most prevalent primary psychiatric diagnoses were: Personality Disorder 22\%, and Schizophrenic Disorder 16,3\%. The most prevalent secondary psychiatric diagnoses were: Personality Disorder 11,9\%, Substance Abuse 10,7\%.

Conclusions: the most prevalent primary and secondary psychiatric diagnosis was personality disorder. The existence of a high level of comorbidity of substance abuse-dependence was recorded. Prisons currently lack Mental Health multidisciplinary teams. This often means that the inmate must be transferred to a hospital with the increased work load and financial cost that this implies.
\end{abstract}

Key words: Mental disorders; antisocial personality disorders; substance-related disorders; prisons. 


\section{INTRODUCCIÓN}

Desde que en la segunda mitad del siglo XX se produjo la transformación de un sistema asistencial basado en el modelo asilar hacia un modelo comunitario, parecería innecesario desde el punto de vista teórico, que existiera una psiquiatría penitenciaria. Sin embargo la realidad es contundente en demostrarnos que los servicios de salud mental comunitarios no están siendo eficaces en el abordaje preventivo, asistencial y rehabilitador de los pacientes graves, especialmente aquellos en situación de pobreza y exclusión social, los cuales tienen mayor riesgo de incurrir en comportamientos delictivos y que están llegando en proporciones crecientes al medio penitenciario.

Por su parte, según describe Hernández Monsal$v^{1}$, el Guardian Newspaper en su edición de 3 de marzo de 2003, señala que en las cárceles estadounidenses hay 300.000 enfermos mentales "porque no tienen otro sitio donde estar", con largas condenas por delitos menores, y que suponen una alta proporción entre los 3.500 presos que esperan en el corredor de la muerte.

El estudio de Gunn ${ }^{2}$ encuentra que el $37 \%$ de los varones condenados en Inglaterra y Gales padecían trastornos psiquiátricos: abuso de sustancias (23\%); trastornos de personalidad (10\%); neurosis (6\%); psicosis $(2 \%)$; y trastornos orgánicos $(0,8 \%)$.

En estudios ingleses más recientes ${ }^{3,4}$ se informa de la presencia de psicosis en el 7,5\% de varones condenados, en el $10 \%$ de varones preventivos y en el $14 \%$ de mujeres (en ambas situaciones). Asimismo el 20\% de los hombres y el $40 \%$ de las mujeres tenían antecedentes de, al menos, un intento autolítico.

Hay que destacar el trabajo de Faze ${ }^{5}$ que revisa la información disponible en 62 estudios ${ }^{6,7,8,9,10}$ en 12 países y que abarca 22.790 prisioneros, que permite establecer las siguientes tasas de prevalencia de enfermedades mentales graves: psicosis (3,7\% de varones y $4 \%$ de mujeres); depresión mayor (10\% de varones y $12 \%$ de mujeres), y trastornos de la personalidad (65\% de varones y $42 \%$ de mujeres). Como se ve estas prevalencias son entre 2 y 4 veces superiores a las encontradas en la población general para psicosis y depresión mayor, suponiendo estos trastornos "tratables" una gran carga asistencial para las instituciones penitenciarias.

Todos los estudios parecen coincidir en que estas proporciones van en aumento en los últimos años ${ }^{11}$.

En nuestro país el estudio Pato ${ }^{12}$ nos da unas tasas de $7,5 \%$ de psicosis, $4,5 \%$ trastornos del humor y $7,5 \%$ trastornos neuróticos, secundarios a situaciones estresantes y somatoformes. Y concluía que sólo un $25 \%$ de la población reclusa no presentaba malestar psíquico.
Objetivo principal:

- Determinar cuáles son los trastornos psiquiátricos principales más prevalentes en los pacientes ingresados en el Módulo Penitenciario a cargo del Servicio de Psiquiatría.

Objetivos secundarios:

- Determinar los diagnósticos psiquiátricos secundarios más frecuentes en estos pacientes.

- Documentar datos sociodemográficos y estancia media.

\section{MATERIAL Y MÉTODO:}

\section{Diseño:}

El estudio que hemos realizado es de tipo descriptivo transversal retrospectivo.

\section{Muestra:}

Formada por todos los pacientes que ingresaron, a cargo del Servicio de Psiquiatría, en el Módulo Penitenciario del H.U.M.S. en el periodo entre el $1 / \mathrm{I} / 2000$ y el $31 / \mathrm{XII} / 2004$, siendo $\mathrm{N}=136$.

\section{Instrumentos:}

- El Servicio de Archivos del Hospital nos ha facilitado el listado de los pacientes que conforman la muestra.

- Se ha llevado a cabo una revisión protocolizada de las historias clínicas, desarrollando un instrumento de recogida de datos, donde constan: datos administrativos (nombre, apellidos, $\mathrm{n}^{\circ}$ historia, estancia media), datos sociodemográficos (edad, sexo), datos clínicos (diagnósticos psiquiátricos principales y secundarios, según criterios CIE-9).

- Los diagnósticos psiquiátricos se han recogido según criterios CIE-9 ya que es el sistema de clasificación que utiliza el Servicio de Archivos de nuestro hospital.

\section{Estadística:}

Se ha realizado estadística descriptiva mediante el programa informático SPSS-12.0 y SPSS-Plus.

\section{RESULTADOS}

1. Durante los 5 años que abarca el estudio, ha habido 836 ingresos totales en el Módulo Penitenciario, de los cuales el $35 \%$ ingresa a cargo del Servicio de Medicina Interna, el 16,2\% a cargo del Servicio de Psiquiatría, el 10,2\% en Digestivo, en el resto de servicios el volumen de pacientes es menor (figura I).

2. De los pacientes psiquiátricos $(\mathrm{N}=136)$, el $90,4 \%$ eran hombres y el $9,6 \%$ mujeres. 
3. La edad media fue de 34,18 años.

4. Más del 50\% de los pacientes estuvieron ingresados menos de 6 días.

5. Los diagnósticos principales más prevalentes fueron: Tr. Personalidad 22\%. Tr. Esquizofrénicos $16,3 \%$. Psicosis afectivas 6,5\%. Dependencia alcohólica 6,5\% (figura II).
6. En la muestra a estudio, se encontró que el $49 \%$ de los pacientes tenían un segundo diagnóstico psiquiátrico, el cual se distribuía de la siguiente manera: Tr. Personalidad 11,9\%. Abuso de drogas 10,7\%. Tr. Esquizofrénicos 5,1\%. Dependencia a drogas 4,5\%. Dependencia a alcohol 4,0\% (figura III).

Porcentaje pacientes Módulo Penitenciario dados de alta pior cada servicio. Hospital Miguel Servet. N: 836

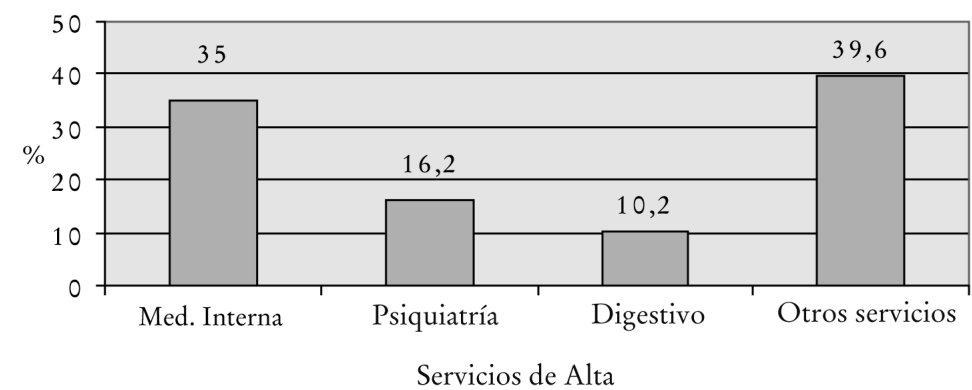

Figura I. Porcentaje de pacientes del Módulo Penitenciario dados de alta por cada Servicio. Hospital Miguel Servet.

Diagnóstico Psiquiátrico principal pacientes ingresados en Módulo Penitenciario Hospital Miguel Servet

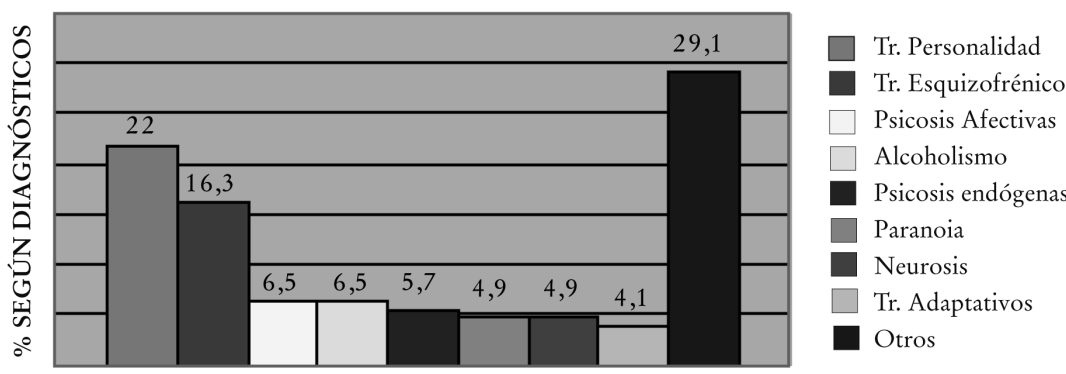

Diagnósticos CIE-9

Figura II. Distribución de los diagnósticos psiquiátricos principales más prevalentes en pacientes del Módulo, ingresados a cargo del Servicio de Psiquiatría. Hospital Miguel Servet.

Diagnóstico Psiquiátrico secundario pacientes ingresados en Módulo Penitenciario Hospital Miguel Servet

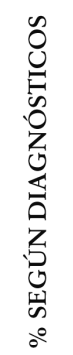
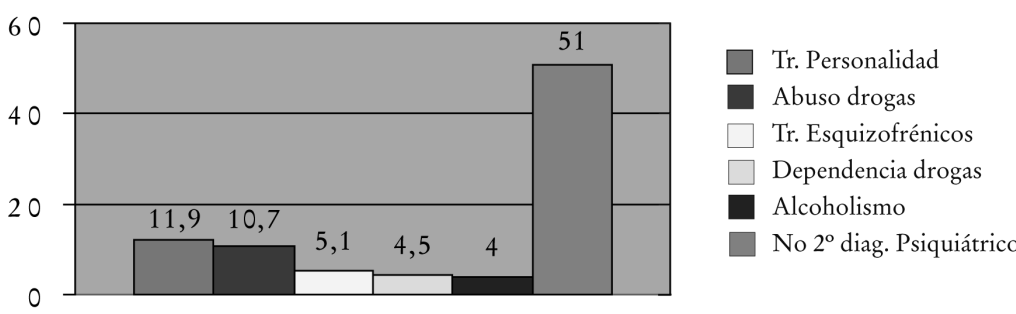

Diagnósticos CIE-9

Figura III. Distribución de los diagnósticos psiquiátricos secundarios más prevalentes en pacientes del Módulo, ingresados a cargo del Servicio de Psiquiatría. Hospital Miguel Servet. 


\section{CONCLUSIONES}

El Servicio de Psiquatría es el que más volumen de pacientes asiste en el Módulo penitenciario, siendo únicamente superado por el Servicio de Medicina Interna.

En nuestro estudio encontramos que el diagnóstico psiquiátrico principal y secundario más prevalente fue el Trastorno de Personalidad y que casi el $20 \%$ de los pacientes presentaba comorbilidad con el abuso-dependencia de tóxicos.

En la actualidad existen pocos estudios sobre el tema y en ellos se documenta el aumento de prevalencia de trastornos psiquiátricos en presos, lo cual justificaría la necesidad de continuar esta línea de investigación.

Las prestaciones asistenciales psiquiátricas que se dispensan en la cárcel son insuficientes e inadecuadas, careciendo de equipos multidisciplinares de Salud Mental, por lo cual en muchas ocasiones se hace necesario el traslado del preso al hospital, suponiendo esto una carga asistencial e incremento de costes.

Por todo ello estudios que determinaran la patología psiquiátrica más prevalente en esta población con características especiales, serían útiles para diseñar modelos de intervención específicos aplicables en los propios centros penitenciarios.

\section{CORRESPONDENCIA}

Hospital Universitario Miguel Servet

Paseo Isabel La Católica, s/n

C.P. 50009 Zaragoza

Correo electrónico: piluca@seny.org

\section{REFERENCIAS BIBLIOGRÁFICAS}

1. Hernández Monsalve M. Pacientes mentales en prisión. Perspectiva internacional. En: La atención a la salud mental de la población reclusa. Madrid: AEN Estudios/30. 2003.
2. Gunn J, Madeu A, Swinton M. Treatment needs of prisioners with psychiatric disorders. BMJ 1991; 303:338-341.

3. Fryers T, Brugha T, Grounds A, Meltzer D. Severe mental illnes in prisoners. BMJ 1998; 317: 10251026 (Editorial).

4. ONS. Psychiatric morbidity among prisoners in England and Wales: En: The prevalence of psychiatric morbidity among adults living en private households. 1997; (www.doh.gov.uk/pub/clocs/ doh/newsurvey 4.pdf).

5. Fazel S, Danesh J. Serious mental disorder in 23.000 prisoners: a systematic review of 62 surveys. Lancet 2002; 359: 545-550.

6. Brinded PM, Simpson AI, Laidlaw TM, Fairley N, Malcom F. Prevalence of psychiatric disorders in New Zealand prisons: a national study. Aust $\mathrm{NZ}$ J Psychiatry. 2001 Apr; 35(2): 166-73.

7. Fazel S, Hope T, O’Donnell I, JacobY R. Hidden psychiatric morbidity in elderly prisoners. $\mathrm{Br} \mathrm{J}$ Psychiatry. 2001 Dec; 179: 535-9.

8. Brooke D, Taylor C, Gunn J, Maden A. Point prevalence of mental disorder in unconvicted male prisoners in England and Wales. BMJ. 1996 Dec 14; 313 (7071): 1524-7.

9. Shaw J, Appleby L, Amos T. Mental disorders and clinical care in people convicted of homicide: national clinical survey. BMJ. 1999 May 8; 318 (7193): 1240-4.

10. Andersen HS, Sestoft D, Lilleback T. A longitudinal study of prisoners on remand: psychiatric prevalence, incidence and psychopathology in solitary vs. Non-solitary confinement. Acta Psychiatr Scand. 2000 Jul; 102 (1): 19-25.

11. Arboleda-Florez J. Mental illness in jail and prisons. Current Opinion in Psychiatry 1999; 12: 677-682.

12. Pato Rodríguez E. Trastornos mentales en reclusos del Centro Penitenciario de Villabona. Principado de Asturias. En: La atención a la salud mental de la población reclusa. Madrid: AEN Estu$\operatorname{dios} / 30$. 